Elsevier required licence: (C) $<2018>$. This manuscript version is made available under the CC-BY-NC-ND 4.0 license http://creativecommons.org/licenses/bync-nd/4.0/ 


\section{Cost Effectiveness of a Community-Delivered Consultation to Improve Infant Sleep Problems and Maternal Well-Being}

Sopany Saing ,MPH ,BCom, BSc(Hons)1, Bonny Parkinson, PhD, MSc, BEc2, Jody Church, MA,BCom1, Stephen Goodall, PhD, MSc, BSc(Hons)1

1Centre For Health Economics Research and Evaluation, University of Technology Sydney, Sydney, New South Wales, Australia;

2Macquarie University Centre for the Health Economy, Macquarie University, Sydney, New South Wales, Australia

\section{Abstract}

\section{Objective}

Post-natal depression is associated with poor infant sleep. The aim of the study was to evaluate the cost effectiveness of a community delivered consultation aimed at improving infant sleep and maternal well-being.

\section{Methods}

A decision analytic model was developed that compared the costs and benefits of an infant sleep consultation versus usual care. The effectiveness of the consultation was based on clinical evidence and improvements in maternal quality of life were estimated by mapping the Edinburgh Postnatal Depression Scale (EPDS) scores to published utility scores. Cost effectiveness was calculated as the incremental cost per quality adjusted life year gained (QALY).

\section{Results}

The statistically significant improvements in mean EPDS scores at 4 and 16 months follow-up were used to estimate the benefit in terms of QALYs. The modelled results demonstrated that the infant sleep consultation is low cost (AUD\$436), more effective in terms of QALYs gained (0.017) and cost effective. The estimated incremental cost-effectiveness ratio was AUD\$4,031 per QALY gained. The main drivers of the model were the use of early parenting centres and nurse training costs.

\section{Conclusions}

Community based nurse delivered infant sleep consultations aid infant sleep, improve maternal quality of life and are cost effective compared to usual care and led to improvements in quality of life through a reduction in postnatal depression. 


\section{Keywords:}

Infant; Sleep Disorder; Depression, Postpartum; Maternal-Child Health Centers; Cost-effectiveness 


\section{Introduction}

Infant sleep problems are common, with $17 \%$ (1) to $46 \%$ (2) of Australian parents reporting problems in the first year postpartum. The most common cause of infant sleep problems are behavioural sleep disorders, such as frequent night waking and difficulty settling to sleep, which may be caused by sleep associations (e.g. rocking, milk or dummy) (3). Less common causes are medical problems such as obstructive sleep apnoea (3).

Infant sleep problems impact adversely on maternal physical and mental-wellbeing $(1,2)$. Postnatal depression, a significant problem in mothers, can be exacerbated by infant sleep problems. In Australia, postnatal depression affects around $16 \%$ of mothers (4) and mothers with clinically significant depression symptoms are often reluctant to accept their diagnosis or seek care (5). Untreated maternal depression can have consequences for both the mother and baby, including a woman's overall well-being, family's functioning, and the child's development (6).

In addition to the health burden, infant sleep problems are directly associated with an increase in health care resource use (7) and indirectly through the treatment of postnatal depression (8). Existing services for women seeking advice on infant sleep problems include GP visits, nurse consultations, paediatrician visits and parenting centres (including day visits, residential stays and home visits). In Australia, these services may be federally funded (Medicare), State funded or privately funded (via private health insurance or out-of-pocket expenses). An analysis of the Longitudinal Study of Australian Children (LSAC) demonstrated that infants with persistent sleep problems are associated with higher Medicare costs (7). Therefore, reducing persistent sleep problems represents an opportunity to leverage existing community health care services and reduce health care costs. 
Three Australian studies have attempted to evaluate the effectiveness of community delivered behavioural infant sleep interventions. These include: the nurse delivered Infant Sleep Study (ISS) cluster randomised controlled trial (RCT)(5); a paediatrician delivered RCT (9); and a before and after study delivered by a single GP or a registered nurse (10). These studies found that short-term consultations significantly reduced infant sleep problems and improved maternal mental health. Internationally, other RCTs have reported reduced prevalence of depression symptoms in mothers and/or improved maternal and infant health outcomes (11-15). Two of these studies were delivered via the internet and focused on the establishment of a bath and bedtime routine $(11,12)$ and two delivered information without a specific behaviour component $(14,15)$. Rickert et al. included a behavioural consultation, however, the only outcome reported was the number of awakening episodes (13).

Only one economic evaluation of a preventative infant sleep problem consultation was identified (16). This study claimed that a behavioural and educational consultation was cost-effective when compared to usual care (from a UK National Health Service Perspective). The reported outcome of this study was 'cost per interruption free night gained', which is difficult to assess true value for money (17). To date, there has been no cost effective analysis of consultations designed to treat infant sleep problems in Australia.

The aim of this study was to conduct an economic evaluation of an infant sleep consultation in the Australian context. In addition to estimating the cost per interruption-free night gained, this study also estimates the incremental cost per quality adjusted life years (QALYs) gained, which enables comparison to other health care interventions and is therefore a more appropriate measure of value for money. This study is also the first study to map changes in the Edinburgh Postnatal Depression Scale to derive utility estimates. We also discuss the pertinent policy implications relating to the different funding mechanisms of health services in Australia. 


\section{Methods}

\section{Systematic review}

A systematic literature review of community delivered infant sleep consultations was undertaken. The search was conducted in EMBASE, PubMed (incorporating Medline), CINAHL, The Cochrane Library and the CRD York databases from inception to 16 October 2014. The search was limited to the English language with no restriction on time.

The search terms included baby, babi*, sleep disorders, infant*, sleep problem*, infant*, sleep disorder* infant* sleep intervention* or behavioral infant sleep intervention* or behavioural infant sleep intervention* or behavioral modification program* or behavioural modification program), maternal-child health centers, community sample or community survey or community-delivered or community-based (both UK and American English). The text words for the consultation were combined using 'OR' and MeSH terms were searched separately, the final results for each group were narrowed down using 'AND'.

Included articles were RCTs or systematic reviews, comparing the effectiveness of a behavioural infant sleep intervention and a suitable comparator in the management of infant sleep problems. The population of interest was mothers and infants (aged 0 to 12 months), and limited to postnatal interventions. Required details included; program information, such as intensity, frequency and who delivered it.

A total of 547 records were identified through the database search, with 524 articles screened based on title and abstract ( 37 duplicates removed and 7 articles identified through other sources) (see Figure 1). The title and abstract review excluded 456 articles with 61 articles included for the full-text review. A total of 38 studies were excluded leaving 23 studies included in the qualitative synthesis, seven systematic literature reviews (18-24), ten unique prevention interventions $(16,25-33)$ and seven unique treatment interventions $(5,9,11-15)$. 
[Insert Figure 1: PRISMA Flow Diagram]

Of the seven treatment studies identified, only two studies fulfilled the study inclusion criteria, the Infant Sleep Study (ISS) and Hiscock et al. (2002) $(5,9,34)$. The treatment studies estimated the effectiveness of a range of interventions, which differed in terms of the behavioural approach and its communication to the parent.

\section{The Infant Sleep Study}

The ISS was a community delivered cluster RCT that assessed outcomes of maternal reports of infant sleep and maternal well-being and examined the delivery of this consultation within an existing health service in Australia. The trial included 49 Maternal and Child Health (MCH) centres (clusters) in Melbourne with 328 mothers (Consultation group $=174$, Control group $=154$ ) (5). The intervention consisted of face-to-face consultations with $\mathrm{MCH}$ nurses, and on average this equated to 1.52 visits of 25 minute duration, for the mothers who received the consultation $(n=100)$. These consultationswere delivered at an infant's visit at eight months of age (one of ten free visits) and the nurses provided mothers with individualised sleep management plans and information handouts about sleep patterns, sleep problems, managing overnight feedings and the use of dummies (pacifiers). (9). Mothers were offered to choose between two behavioural interventions controlled crying or camping out, and bedtime routines

In the 'controlled crying' approach, parents were instructed to respond to their infant's crying by increasing the time intervals to allow the child to learn to self-settle $(5,9)$. In the 'camping out' approach, the parent sat with the child as they learnt to independently fall asleep $(5,9)$. The effectiveness of the ISS study estimated maternal well-being using the mean change in the Edinburgh Postnatal Depression Scale (EPDS) score, measured when the infant was 10 and 12 months (5) and at two years of age (34).Further details of the trial are described elsewhere $(5,34$ - 
37). The Department of Education and Early Childhood Development (DEECD) have since trained all Victorian MCH Nurses in the program (38).

Hiscock et al. (2002) was an RCT of 156 mothers (78 each in the consultation and control group) with an intervention similar to that in the ISS study (9), but differing in the inclusion of three consultations (instead of one) that were provided by a paediatrician (rather than nurses) (9). In addition to this, participants were only followed until the infants reached 12 months of age; hence, outcomes were not comparable across studies. As the data could not be pooled, additional sensitivity analyses were conducted using results from Hiscock et al. (2002).

\section{Cost-effectiveness model}

A decision analytic model (built in Microsoft $\operatorname{Excel}^{\circledR}, 2010$ ) was constructed to estimate the cost effectiveness of an infant sleep consultation versus usual care for women attending $\mathrm{MCH}$ centres. The outcome was the incremental cost per QALY gained derived from the EPDS score. A health care perspective was applied in the economic evaluation, taking into account the consultation, training costs and health care resource costs. The time horizon for the model was 16 months (based on the follow-up in the trial), however results were also presented for four months follow-up.

\section{Measurement of Effectiveness}

The primary outcome measured in the clustered RCT was the maternal report of infant sleep ("Over the last 2 weeks, has your baby's sleep generally been a problem for you? Yes/No"). For this study the secondary outcome measure, mean EPDS score, was used for the model (Table 1). The study found that the mothers in the intervention group had lower mean EPDS scores than the control group at both 10 and 12 months of age(5). Based on the mean EPDS score, linear interpolation was used to plot the EPDS score at two, four and 16 months follow-up. A previous study by Stevenson et al (2010) was used to map the change in EPDS score to the change in utility. The authors plotted the change in EPDS scores from a cognitive behavioural therapy trial against the individual SF-6D utility 
scores from women in the PONDER trial (39). An estimated regression equation ( $y=-0.0113 x+$ $0.0625)$ was used to transform the gain in EPDS into utility values $(40,41)$. We used the same 'beta coefficient' and the intercept was adjusted for the 20 week trial period to ensure it was consistent with an annual utility (41). Baseline utility scores were derived from a study in of 623 postnatal women based on the SF-36 [0.809 (95\% Cl: 0.796, 0.822)] (42). These changes in utility scores were converted into QALYs. Outcomes measured at the 16-month follow-up were discounted at an annual rate of $5 \%$.

[Insert Table 1: model inputs for base case and sensitivity analysis]

\section{Estimation of Resources use and Costs}

Resource use collected during the ISS trial (5) was used to estimate costs (calculated in 2014-2015 prices) (Table 2). The costs of delivering the consultation were based from the ISS trial (which reported the average number of visits and consultation length) and the written material provided to the mothers. The total average cost per participant was estimated to be $\$ 13.39$ for the consultation group and $\$ 4.47$ for the control group. Training costs for the nurses were included in the base case (5 hours of training by a paediatrician, child psychologist and the nurse time equating to $\$ 213.75$ ). Training costs were multiplied by the $\mathrm{MCH}$ centre to mother ratio ( $25 \mathrm{MCH}$ centres for 174 participants) and nurse costs were multiplied by the nurse to mother ratio ( 38 nurses for 174 participants). This ratio was adjusted to test the impact of economies of scale in training (scenario analysis).

Mothers in the ISS trial reported health care resource use during the four-month follow-up period. Health care resource use was based on the four month follow-up data only and not extrapolated to a 16 month time horizon. In addition, an alternative base case was presented for a four month time horizon (5). These services included day stays or residential stays at early parenting centres, nurse telephone support, nurse visits, general practitioner (GP) visits, and paediatrician consultations. The 
only significant difference in resource use between the consultation and control group was residential stays at parenting centres $(\$ 3,390$ per night). The base case assumed lower residential stays in the consultation group (odds ratio $=0.311, p=0.0498$ ) at the two month follow-up but not at the four month follow-up, leading to a cost saving over the four months of $\$ 170$. The average resource use across both groups was used for the other health care services and was estimated to be $\$ 32$. Pharmaceutical costs or direct maternal health care costs were not included, however it was assumed that the GP visits would include a portion of these costs.

[Insert Table 2: Cost parameters used in the model]

\section{Sensitivity analysis}

Univariate sensitivity analysis was undertaken using the upper and lower $95 \%$ confidence interval to test parameter uncertainty in the model. Probabilistic sensitivity analysis was conducted to test the joint uncertainty across all model parameters. Beta distributions were applied to probabilities, gamma distributions to utilities and log normal distributions to odds ratios (control group resource use). Distributions were applied to all parameters and Monte Carlo simulation was conducted $(10,000$ iterations $)$

The following scenario analyses were undertaken: 1) no difference in health care resource use apart from the cost of the consultation; 2) health care resource use based on use within the trial regardless of statistical significance; 3) shorter nurse consultation; 4) health care resource use extrapolated for the remaining 12 months; 5) exclusion of training costs; 6) higher training costs; 7) only women with EPDS $\geq 10$ included for more severe symptoms; and 8) non-significant 4 month follow-up EPDS score from Hiscock et al. 2002 (9).

\section{Results}

\section{Base case results}


Table 3 presents the estimated incremental cost per QALY gained in the consultation group compared to the control group over a time horizon of 16 months. In the base case, training costs and reduced residential stays at the early parenting centre in the consultation group were included. The consultation group had a higher incremental cost $(\$ 68)$ due to costs in delivering the consultation and associated training. There was an incremental gain of 0.017 QALYs (1.4 versus 1.38 ) in the consultation group compared to the control group, resulting in an ICER of $\$ 4,031$ per QALY gained in the base case.

\section{One-way sensitivity analysis}

The one-way sensitivity analysis shown in the tornado diagram (Figure 2), demonstrates that the model was most sensitive to the probability of overnight residential stays. Increasing the probability of overnight stays in the consultation group (increasing the odds ratio from 0.311 to 0.999 ) at the two-month follow-up increased the ICER to approximately $\$ 14,000$ per QALY gained. Further, increasing the baseline EPDS mean score in the control group increased the ICER to approximately $\$ 24,000$ per QALY gained. Increasing the overnight stay proportion in the control group (two month follow-up) to 0.12 from 0.075 made the consultation group cost saving. The model was robust to one-way changes in resource use, low and high estimates in the efficacy variables, and mean EPDS score at different follow-up periods.

[Insert Figure 2: Tornado diagram for one-way sensitivity analysis]

\section{Probabilistic sensitivity analysis}

The probabilistic analysis showed that the consultation group was the most cost effective strategy when the willingness-to-pay threshold was higher than $\$ 3,055$ per QALY gained. Assuming a threshold of $\$ 50,000$ per QALY gained (given there is no explicit threshold in Australia); there was a $97 \%$ probability that the consultation was cost effective relative to usual care.

\section{Scenario analysis}


The consultation group became the dominant option (less costly, more effective) under scenarios of resource use where the proportions were based on those in the trial (regardless of statistical significance, favouring the consultation group), exclusion of training costs and extrapolation of resource use for the 16-month follow-up. Similarly, the ICER was lower (i.e. became more favourable for the consultation group) in a scenario with higher baseline EPDS scores, reflecting a situation where women with more severe postnatal depression symptoms were treated. Alternatively, when the overnight residential stay use was assumed to be the same in both groups (along with other health care resource use), the ICER increased to $\$ 14,209$ per QALY gained, indicating that this resource use difference is a driver in the model.

[Insert Table 3: Cost-effectiveness results for base case and scenario analysis of consultation and control group, 16 month follow-up (discounted) and 4 month follow-up (not discounted)]

\section{Discussion}

The study demonstrated that an infant sleep consultation, as described in the ISS study, was cost effective. The model was sensitive to assumptions regarding health care resource use, in particular, the utilisation of residential stays at early parenting centres. However, these conclusions are robust, in that ISS was likely to be cost effective across all parameter values and under various scenarios.

These results are congruent with an economic evaluation of a preventative infant sleep consultations (16). The authors considered both behavioural and educational consultations to be cost effective, with an incremental cost of GBP£0.56 ( \$AUD 1.70) and GBP£4.13 ( $\sim$ SAUD 12.51) per interruption free night gained, respectively (17). However, decision makers are likely to find it difficult to interpret the 'cost per interruption free night gained' in terms of value for money relative to other health service. For comparison, using the four-month follow-up data for the proportion of infants with existing parent-reported infant sleep problems (5) (based on the same costs used in the analysis), the cost per infant sleep problem avoided was $\$ 422$. This highlights the difficulties in 
comparing such outcomes. A strength of this study is that the health gains are reported in terms of QALYs, which are more readily comparable across consultations and disease areas. Another key strength of this study is that effectiveness data was based on a large cluster RCT. As infant sleep problems and EPDS scores are likely to improve over time, prospective RCT data can capture this and can control for other confounding factors compared to before and after studies (43).

Currently, the $\mathrm{MCH}$ Key Ages and Stages (KAS) Consultations are delivered by local government, with the State Government (DEECD) and local government each funding 50 per cent of the cost (44). Additional consultations (outside of the 10 KAS) are not funded by the DEECD (45). The infant sleep consultation could be provided within the eight month and 12 month visits without adding additional costs (5). In Victoria there is complete State and Local Government funding for the $\mathrm{MCH}$ program. Therefore, mechanisms are currently in place to introduce sleep consultations to the wider population in Victoria. The out of pocket expenses that could be incurred by women seeking help for their infant sleep problems have not been included in this economic evaluation. The incorporation of the consultation at one of the key ages and stages could reduce out of pocket expenses for the mothers (if seeking help from GPs or paediatricians) and possible downstream health resource costs. This program represents an opportunity to provide services that would be accessible to all women.

All the women in the study had infants with self-reported infant sleep problems. Therefore, at baseline, the median EPDS score was 8 in both the consultation and control group and the proportion of women with an EPDS score $>9$ were $42 \%$ and $38 \%$, respectively (5). A limited number of mothers reported high depression scores (EPDS $\geq 13$ ). This may limit the generalisability of the results to mothers with severe postnatal depression. Further, the consultation did not result in statistically significant improvements in women with an EPDS > 9 compared to control at the two and four month follow-up, but was statistically significant at 16 months $(5,34)$.

A limitation of this study is that utility scores used in the model were from a different postnatal population, due to the unavailability of individual-level utility data from the ISS RCT $(5,40)$. The 
validity of translating changes from the EPDS score to changes in SF-6D has not been tested.

Mothers completed SF-12 questionnaires and statistically significant improvements in physical and mental health were reported after the two and four month follow-ups (5). Thus, there is support that the consultation improved the health related quality of life of mothers, however, SF-12 scores cannot be converted into utility values without patient level data. If individual-level utility data were available, there would be no need to assume exchangeability of populations of 'postnatal women' at different times after birth. Although the model mapped the values directly, the ISS RCT recruited Australian postnatal women at 7 months after birth(5) compared to a postnatal UK population, measured at 6 weeks after birth (40).

With respect to costs, there were several limitations. First, resource use was based on participant's self-reports and may be subject to recall bias. However, assuming that that there is no difference in resource use in the consultation and control groups resulted in an increased cost of $\$ 14,209$ per QALY gained. Second, the costs of treating postnatal depression symptoms were not included in the model due to a lack of information about any associated resource use. Third, while upfront training costs were included in the base case, ongoing training costs were not included as the exact timing of this training was uncertain. In practice, new nurses would need to be trained to deliver the consultation and existing nurses would require refresher training every few years. The costs were based on the resources used in the trial, however currently the costs for nurses to take the training (either online $(\$ 50)$ or face-to-face ( $\$ 145$ Victoria only) is run by The Royal Children's Hospital Melbourne (46). These costs are lower than the estimated costs used in the model, and would favour the consultation, resulting in a better cost-effectiveness ratio (holding all other variables constant).

Finally, participation in the eight month $\mathrm{MCH}$ visit was $85.5 \%$ in State-wide Victoria for all families in 2014 (47). In contrast, the uptake of the consultation in the trial was moderate (57\%) by families in the consultation group despite all reporting an infant sleep problem (34). There may be a higher 
uptake of the consultation if it forms part of the wider $\mathrm{MCH}$ program, leading to greater health benefits. Although the study was conducted in Victoria, the results are generalisable in Australia, as each state currently funds early childhood centres where this type of intervention could be implemented. The generalisability of these findings internationally is dependent on the current services available in each country.

This is the first Australian study to evaluate the cost effectiveness of a community based nurse delivered infant sleep consultation. The results show that the intervention is cost-effective compared to usual care. However, the results may only be applicable to mothers with mild depression. 


\section{Conflict of interest}

The authors declare that they have no conflicts of interest.

\section{References}

1. Martin J, Hiscock, H., Hardy, P., Davey, B., and Wake, M. Adverse associations of infant and child sleep problems and parent health: An Australian population study. Pediatrics. 2007;119(5):947-55.

2. Hiscock $\mathrm{H}$, and Wake, M. Infant sleep problems and postnatal depression: A community-based study. Pediatrics. 2001;107(6):1317-22. 3. Waters KA, Suresh, S., and Nixon, G. M. Sleep disorders in children. MJA. 2013;199:S31-S5.

4. Buist A and Bilszta J. The beyondblue national postnatal screening program, prevention and early intervention 2001-2005, final report. Vol 1: National screening program. Melbourne: beyondblue: the national depression initiative. 2006.

5. Hiscock H, Bayer J, Gold L, et al. Improving infant sleep and maternal mental health: A cluster randomised trial. Archives of Disease in Childhood. 2007 November 1, 2007;92(11):952-8.

6. Sontag-Padilla L, Schultz D, Reynolds K, et al. Maternal depression: Implications for systems serving mother and child. RAND Corporation, 2013.

7. Quach J, Gold L, Hiscock H, et al. Primary healthcare costs associated with sleep problems up to age 7 years: Australian population-based study. BMJ Open. 2013 January 1, 2013;3(5).

8. Deloitte Access Economics. The cost of perinatal depression in Australia: Final report. Postal and antenatal depression association. 2012. . 9. Hiscock H, Wake M. Randomised controlled trial of behavioural infant sleep intervention to improve infant sleep and maternal mood. British Medical Journal. 2002;324(7345):1062-5.

10. Symon B, Bammann M, Crichton G, et al. Reducing postnatal depression, anxiety and stress using an infant sleep intervention. BMJ Open. 2012 January 1, 2012;2(5).

11. Mindell JA, Du Mond CE, Sadeh A, et al. Efficacy of an internet-based intervention for infant and toddler sleep disturbances. Sleep. 2011 Apr;34(4):451-8.

12. Mindell JA, Telofski LS, Wiegand B, et al. A nightly bedtime routine: Impact on sleep in young children and maternal mood. Sleep. 2009 May;32(5):599-606. 
13. Rickert VI, Johnson CM. Reducing nocturnal awakening and crying epi sodes in infants and young children. A comparison between scheduled awakenings and systematic ignoring. Pediatrics. 1988;81(2):203-12.

14. Scott G, Richards MPM. Night waking in infants: Effects of providing advice and support for parents. J Child Psychol Psychiatry. 1990;31:551-67. 15. Seymour FW, Brock P, During M, et al. Reducing sleep disruptions in young children: Evaluation of therapistguided and written information approaches: A brief report. J Child Psychol Psychiatry. 1989;30:913-8.

16. Sleep JG, P. St James-Roberts, I. and Morris, S. A randomized controlled trial to compare alternative strategies for preventing infant crying and sleep problems in the first 12 weeks: The cosi study. Primary Health Care Research and Development. 2002;3:176-83.

17. Morris S, St James-Roberts I, Sleep J, et al. Economic evaluation of strategies for managing crying and sleeping problems. Archives of Disease in Childhood. 2001 January 1, 2001;84(1):15-9.

18. Douglas PS, and Hill. P.S. Behavioral sleep interventions in the first six months of life do not improve outcomes for mothers or infants: A systematic review. Journal of Developmental and Behavioral Pediatrics. 2013;34(7):497-507.

19. Bryanton J, Beck, C.T. Postnatal parental education for optimizing infant general health and parent-infant relationships. Cochrane Database of Systematic Reviews. 2013.

20. Yonemoto N, Dowswell T, Nagai S, et al. Schedules for home visits in the early postpartum period. The Cochrane database of systematic reviews. 2013;7:CD009326.

21. Barlow J, Smailagic N, Huband N, et al. Group-based parent training programmes for improving parental psychosocial health. The Cochrane database of systematic reviews. 2014;5:CD002020.

22. Dennis CL, Dowswell T. Psychosocial and psychological interventions for preventing postpartum depression. The Cochrane database of systematic reviews. 2013;2:CD001134.

23. Ramchandani P, Wiggs L, Webb V, et al. A systematic review of treatments for settling problems and night waking in young children2000 2000-01-22 08:00:00. 209-13 p.

24. Mindell JA KB, Lewin DS, Meltzer, LJ and Sadeh A. Behavioral treatment of bedtime problems and night wakings in infants and young children. Sleep. 2006;29:1263-76.

25. Hiscock H, Cook F, Bayer J, et al. Preventing early infant sleep and crying problems and postnatal depression: A randomized trial. Pediatrics. 2014 January 6, 2014;133.

26. Kerr SM, Jowett SA, Smith LN. Preventing sleep problems in infants: A randomized controlled trial. J Adv Nurs. 1996 Nov;24(5):938-42. 
27. Pinilla T BL. Help me make it through the night: Behavioral entrainment of breast-fed infants' sleep patterns. Pediatrics 1993;91:43644.

28. St James-Roberts I, Sleep J, Morris S, et al. Use of a behavioural programme in the first 3 months to prevent infant crying and sleeping problems. Journal of paediatrics and child health. 2001 Jun;37(3):289-97. 29. Stremler R, Hodnett E, Kenton L, et al. Effect of behaviouraleducational intervention on sleep for primiparous women and their infants in early postpartum: Multisite randomised controlled trial2013 2013-0320 11:42:06.

30. Stremler R, Hodnett E, Lee K, et al. A behavioral-educational intervention to promote maternal and infant sleep: A pilot randomized, controlled trial. Sleep. 2006 Dec;29(12):1609-15.

31. Symon B, Marley, J.E., Martin, J.A., and Norman, E.R. Effect of a consultation teaching behaviour modification on sleep performance in infants: A randomised controlled trial. MJA. 2005;182(215).

32. Wolfson A LP, Futterman A. Effects of parent training on infant sleeping patterns, parents' stress, and perceived parental competence. Journal of Consulting and Clinical Psychology. 1992;60(1):41-8.

33. Christie J BB. The effect of health visitors' postpartum home visit frequency on first-time mothers: Cluster randomised trial. International Journal of Nursing Studies. 2011;48(6):689-702.

34. Hiscock H, Bayer JK, Hampton A, et al. Long-term mother and child mental health effects of a population-based infant sleep intervention: Cluster-randomized, controlled trial. Pediatrics. 2008 September 1, 2008;122(3):e621-e7.

35. Wake M, Price A, Clifford S, et al. Does an intervention that improves infant sleep also improve overweight at age 6? Follow-up of a randomised trial. Arch Dis Child. 2011;96:526-32.

36. Price A, Wake M, Ukoumunne OC, et al. Five-year follow-up of harms and benefits of behavioural infant sleep intervention: Randomised trial. Paediactrics. 2012;130(4).

37. Price AMH, Wake M, Ukoumunne OC, et al. Outcomes at six years of age for children with infant sleep problems: Longitudinal community-based study. Sleep medicine. 2012;13:991-8.

38. The Royal Children's Hospital Melbourne. Sleep studies - infant sleep study (iss) 2015 [cited 201522 May]. Available from: http://www.rch.org.au/ccch/research-projects/Infant Sleep Study/.

39. Stevenson MD, Scope A, Sutcliffe PA, et al. Group cognitive behavioural therapy for postnatal depression: A systematic review of clinical effectiveness, cost-effectiveness and value of information analyses. Health Technol Assess. 2010 Sep;14(44):1-107, iii-iv. 
40. Morrell C J, Warner R, Slade P, et al. Psychological interventions for postnatal depression: Cluster randomised trial and economic evaluation. The ponder trial. Health Technology Assessment. 2009 2009/07/10;13(30):176.

41. Stevenson MD, Scope A, Sutcliffe PA. The cost-effectiveness of group cognitive behavioral therapy compared with routine primary care for women with postnatal depression in the UK. Value in Health. 2010;13(5). 42. Petrou S, Morrell J, Spiby H. Assessing the empirical validity of alternative multi-attribute utility measures in the maternity context. Health and Quality of Life Outcomes. 2009;7(1):40.

43. Grimshaw J, Campbell M, Eccles M, et al. Experimental and quasiexperimental designs for evaluating guideline implementation strategies. Family practice. 2000 Feb;17 Suppl 1:S11-6.

44. State Government of Victoria, Department of Education and Early Childhood Development. The state of victoria's children 2012: Early childhood. Melbourne: Department of Educationand Early Childhood Development, 2013.

45. State Government of Victoria, Department of Education and Early Childhood Development, Municipal Association of Victoria. Maternal and child health service funding: Information sheet - april 2009. In: Department of Education and Early Childhood Development, editor. Melbourne2009. 46. RACGP. Behavioural intervention: Infant sleep problems and maternal mood. Royal Australian College of General Practitioners, editor2014.

47. State Government of Victoria, Department of Education and Training. Maternal \& child health services annual report: Statewide 2013-2014. Melbourne: Department of Education and Training, 2014.

48. Fair Work Australia. Nurses and midwives (victorian public sector) (single interest employers) enterprise agreement 2012-2016. 2012. 49. Officeworks. 2015 [cited 201515 June]. Available from:

http://www.officeworks.com.au/print/print-and-copy/self-service/selfservice-copies.

50. AMA Victoria. Specialists in public hospitals - rates of pay 2015 [cited 20156 July]. Available from:

https://amavic.com.au/page/Specialists in Public Hospitals/Public Hospit al Working Conditions/Rates of pay/\#1.

51. State Government of Victoria, Department of Health \& Human Services. Volume 3 human services policy and funding plan 2015-18, chapter 2: Human services output budget information and unit prices Melbourne: Department of Health \& Human Services, 2015.

52. Australian Goverment Department of Health. Mbs online 2015 [cited 20156 July]. Available from: http://www9.health.gov.au/mbs/search.cfm. 
Figure 1: PRISMA Flow Diagram for systematic literature review of community delivered infant sleep consultations

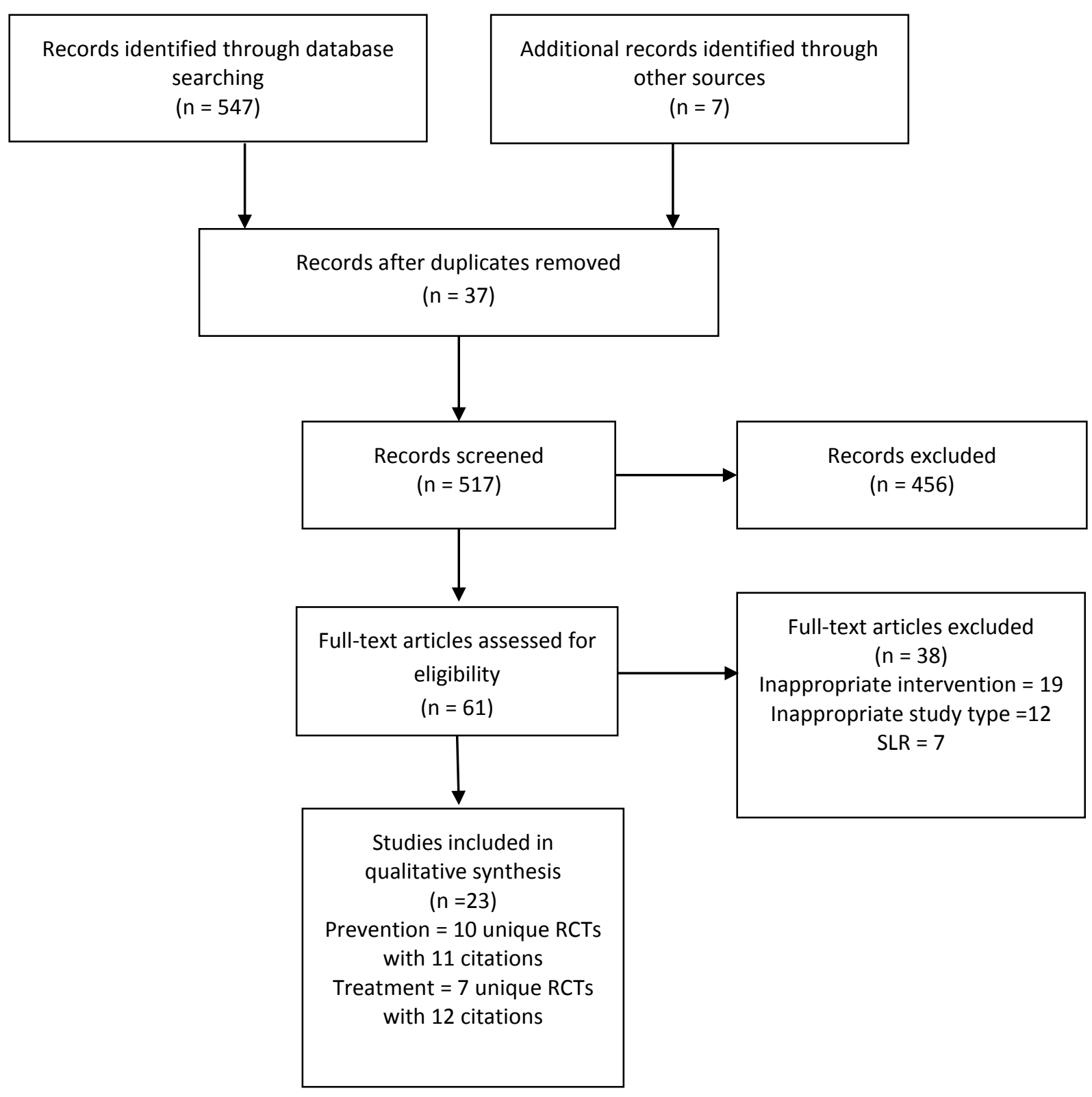

Source: adapted from Moher D, Liberati A, Tetzlaff J, Altman DG, The PRISMA Group (2009).

Preferred Reporting Items for Systematic Reviews and Meta-Analyses: The PRISMA Statement. PLoS Med 6(6): e1000097. doi:10.1371/journal.pmed1000097 
Figure 1: Tornado diagram for one-way sensitivity analysis

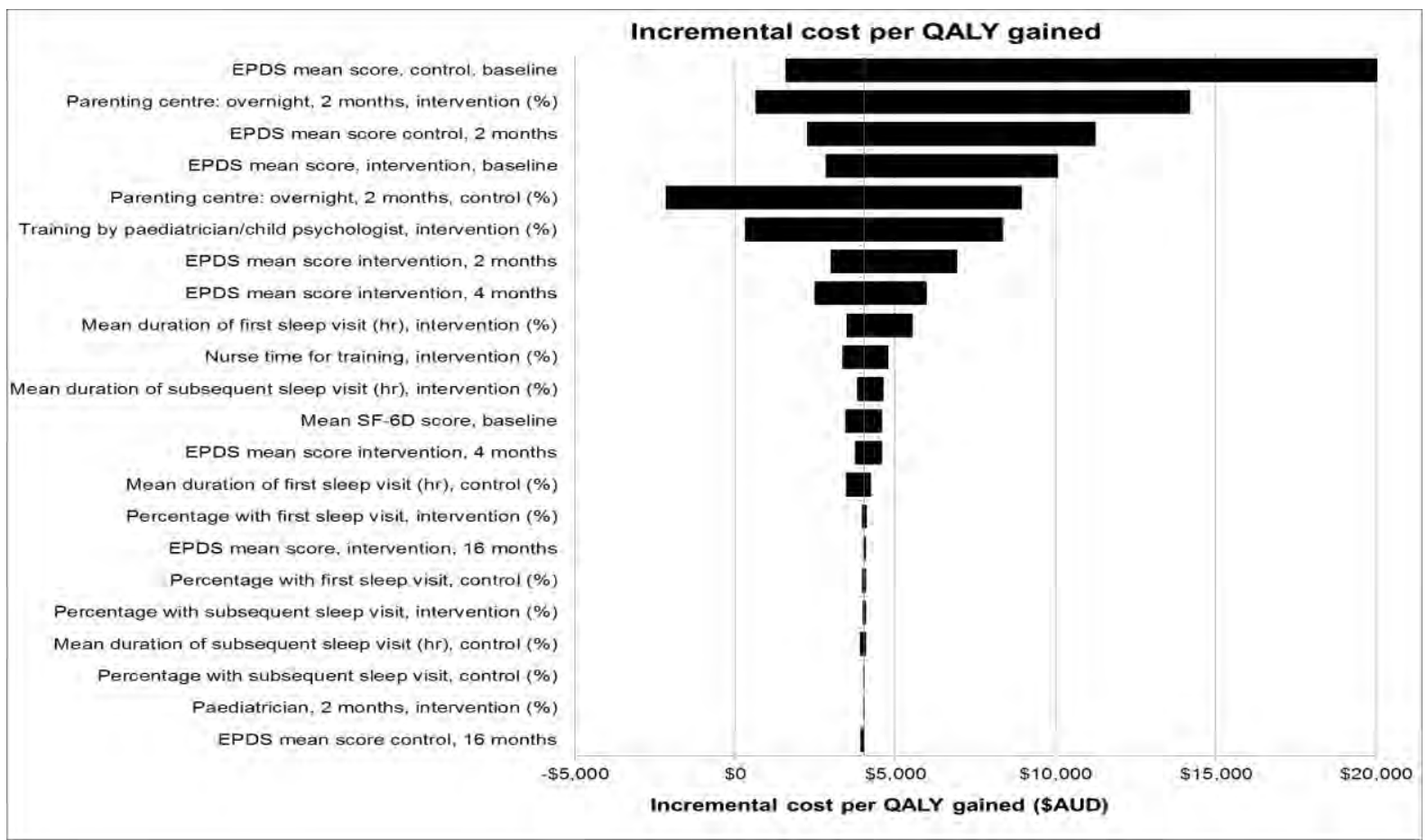


Table 1: model inputs for base case and sensitivity analysis

\begin{tabular}{|c|c|c|c|c|c|}
\hline Variable & Value (SE) & $a^{2}$ & $\beta / \lambda^{2}$ & Distribution & Source \\
\hline \multicolumn{6}{|l|}{ Efficacy (EPDS mean score) } \\
\hline Intervention, baseline & $8.40(0.40)$ & 437.08 & 0.02 & Gamma & \multirow{6}{*}{ ISS study(5) } \\
\hline Control, baseline & $8.40(0.41)$ & 417.77 & 0.02 & Gamma & \\
\hline Intervention, 2 months & $6.80(0.39)$ & 298.67 & 0.02 & Gamma & \\
\hline Control, 2 months & $7.80(0.45)$ & 303.70 & 0.03 & Gamma & \\
\hline Intervention, 4 months & $5.90(0.37)$ & 253.82 & 0.02 & Gamma & \\
\hline Control, 4 months & $7.20(0.42)$ & 289.49 & 0.02 & Gamma & \\
\hline Intervention, 16 months & $5.50(0.35)$ & 241.76 & 0.02 & Gamma & \multirow{2}{*}{16 month follow-up(34) } \\
\hline Control, 16 months & $6.72(0.46)$ & 217.95 & 0.03 & Gamma & \\
\hline \multicolumn{6}{|l|}{ Utility (SF-6D score) } \\
\hline Baseline & $0.81(0.01)$ & 3143.47 & 742.15 & Beta & \multirow{2}{*}{ Petrou (2009)(42) } \\
\hline EPDS $\geq 10$, baseline & $0.72(0.01)$ & 2176.36 & 829.66 & Beta & \\
\hline Regression beta coefficient & $-0.0113(0.00)$ & -0.01 & 0.00 & Log normal & Stevenson (2010)(39) $5 \mathrm{mth}$ \\
\hline \multicolumn{6}{|c|}{ Resource use (all from ISS study)(5) - Consultation } \\
\hline \multicolumn{6}{|c|}{ MCH nurse consultation (proportions) } \\
\hline First visit & $0.57(0.04)$ & 99.43 & 73.57 & Beta & 100/174 families, $25 \mathrm{~min}$ \\
\hline Subsequent visit & $0.30(0.03)$ & 51.70 & 121.30 & Beta & 1.52 visits $^{1}, 19 \mathrm{~min}$ \\
\hline Training & $0.14(0.03)$ & 24.86 & 148.14 & Beta & 25/174 families \\
\hline Nurse time for training & $0.22(0.03)$ & 37.78 & 135.22 & Beta & 38/174 families \\
\hline \multicolumn{6}{|c|}{ Non-MCH nurse professional health care (Intervention compared to control) - 8-10 months } \\
\hline Parenting centre: day & $0.50(0.64)$ & -0.69 & 0.64 & Log normal & \multirow{6}{*}{$\begin{array}{l}\text { OR calculated from reported } \\
\text { events from ISS study }\end{array}$} \\
\hline Parenting centre: overnight & $0.31(0.60)$ & -1.17 & 0.60 & Log normal & \\
\hline Telephone support & $0.60(0.92)$ & -0.52 & 0.92 & Log normal & \\
\hline Nurse visit & $0.13(1.52)$ & -2.07 & 1.52 & Log normal & \\
\hline Family doctor & $0.59(0.54)$ & -0.53 & 0.54 & Log normal & \\
\hline Paediatrician & $0.60(0.92)$ & -0.52 & 0.92 & Log normal & \\
\hline \multicolumn{6}{|c|}{ Non-MCH nurse professional health care (Intervention compared to control) - 10-12 months } \\
\hline Parenting centre: day & $0.67(0.77)$ & -0.41 & 0.77 & Log normal & \multirow{6}{*}{$\begin{array}{l}\text { OR calculated from reported } \\
\text { events from ISS study }\end{array}$} \\
\hline Parenting centre: overnight & $0.67(0.77)$ & -0.41 & 0.77 & Log normal & \\
\hline Telephone support & $0.89(0.64)$ & -0.11 & 0.64 & Log normal & \\
\hline Nurse visit & $0.17(1.10)$ & -1.75 & 1.10 & Log normal & \\
\hline Family doctor & $0.63(0.60)$ & -0.46 & 0.60 & Log normal & \\
\hline Paediatrician & $0.29(1.16)$ & -1.22 & 1.16 & Log normal & \\
\hline \multicolumn{6}{|c|}{ Resource use - Control } \\
\hline \multicolumn{6}{|c|}{ MCH nurse consultation (proportions) } \\
\hline First visit & $0.22(0.03)$ & 38.19 & 134.81 & Beta & 34/154 families, $25 \mathrm{~min}$ \\
\hline Subsequent visit & $0.07(0.02)$ & 12.22 & 160.78 & Beta & 1.32 visits $^{1}, 19 \mathrm{~min}$ \\
\hline \multicolumn{6}{|c|}{ Non-MCH nurse professional health care (Intervention compared to control) - 8-10 months } \\
\hline Parenting centre: day & $0.05(0.02)$ & 6.95 & 139.05 & Beta & \multirow{6}{*}{ Proportions } \\
\hline Parenting centre: overnight & $0.07(0.02)$ & 10.93 & 135.07 & Beta & \\
\hline Telephone support & $0.02(0.01)$ & 2.98 & 143.02 & Beta & \\
\hline Nurse visit & $0.02(0.01)$ & 3.48 & 142.52 & Beta & \\
\hline Family doctor & $0.06(0.02)$ & 8.94 & 137.06 & Beta & \\
\hline Paediatrician & $0.02(0.01)$ & 2.98 & 143.02 & Beta & \\
\hline \multicolumn{6}{|c|}{ Non-MCH nurse professional health care (Intervention compared to control) - 10-12 months } \\
\hline Parenting centre: day & $0.03(0.01)$ & 3.97 & 141.03 & Beta & \multirow{2}{*}{ Proportions } \\
\hline Parenting centre: overnight & $0.03(0.01)$ & 3.97 & 141.03 & Beta & \\
\hline
\end{tabular}




$\begin{array}{lllll}\text { Telephone support } & 0.03(0.02) & 4.97 & 140.03 & \text { Beta } \\ \text { Nurse visit } & 0.03(0.02) & 4.97 & 140.03 & \text { Beta } \\ \text { Family doctor } & 0.05(0.02) & 6.95 & 138.05 & \text { Beta } \\ \text { Paediatrician } & 0.02(0.01) & 2.98 & 142.02 & \text { Beta }\end{array}$

${ }^{1}$ Assumption that only a single subsequent visit was received. $\mathrm{SE}=$ Standard Error. $\mathrm{Cl}=$ Confidence interval.

${ }^{2}$ Log normal distribution has a shape parameter $\sigma$ and scale parameter $\mu$.

Abbreviations: min, minutes 
Table 2: Cost parameters used in the model

\begin{tabular}{|c|c|c|c|}
\hline Variable & Value & Calculation & Source \\
\hline \multicolumn{4}{|c|}{$\mathrm{MCH}$ nurse consultation - consultation delivery } \\
\hline $\mathrm{MCH}$ visit, per hour & $\$ 39.12$ & $\$ 1,369.20$ pw / 35 hours pw & $(5,48)$ \\
\hline Written material & $\$ 0.32$ & $\$ 0.08$ per handout * 4 handouts & $(5,48,49)$ \\
\hline \multicolumn{4}{|c|}{ Nurse training program costs for consultation } \\
\hline Paediatrician's time & $\$ 648.53$ & $(\$ 4,539.70 \mathrm{pw} / 35$ hours pw $) * 5$ hrs & (50) \\
\hline Child psychologist time & $\$ 648.53$ & $(\$ 4,539.70 \mathrm{pw} / 35$ hours pw $)$ * 5 hrs & (50) \\
\hline Nurse time & $\$ 195.60$ & $\$ 39.12$ per hr * 5 hours & (48) \\
\hline \multicolumn{4}{|l|}{ Other health care resource use } \\
\hline Parenting centre: overnight & $\$ 3,390.00$ & Activity ID 31256, residential core & (51) \\
\hline Parenting centre: day visit & $\$ 401.79$ & Activity ID 31256, day stay & (51) \\
\hline Telephone support, per half $\mathrm{hr}$ & $\$ 19.56$ & $(\$ 1,369.20 \mathrm{pw} / 35$ hours pw $) * 0.5 \mathrm{hrs}$ & (48) \\
\hline Nurse visit, per hour & $\$ 39.12$ & (\$1,369.20 pw / 35 hours pw) & (48) \\
\hline Family doctor, per visit & $\$ 37.05$ & Full fee, MBS 23 & (52) \\
\hline Paediatrician, per visit & $\$ 85.55$ & Full fee, MBS 104 & (52) \\
\hline
\end{tabular}

Abbreviations: hr, hour; pw, per week 
Table 3: Cost-effectiveness results for base case and scenario analysis of consultation and control group, 16 month follow-up (discounted) and 4 month follow-up (not discounted)

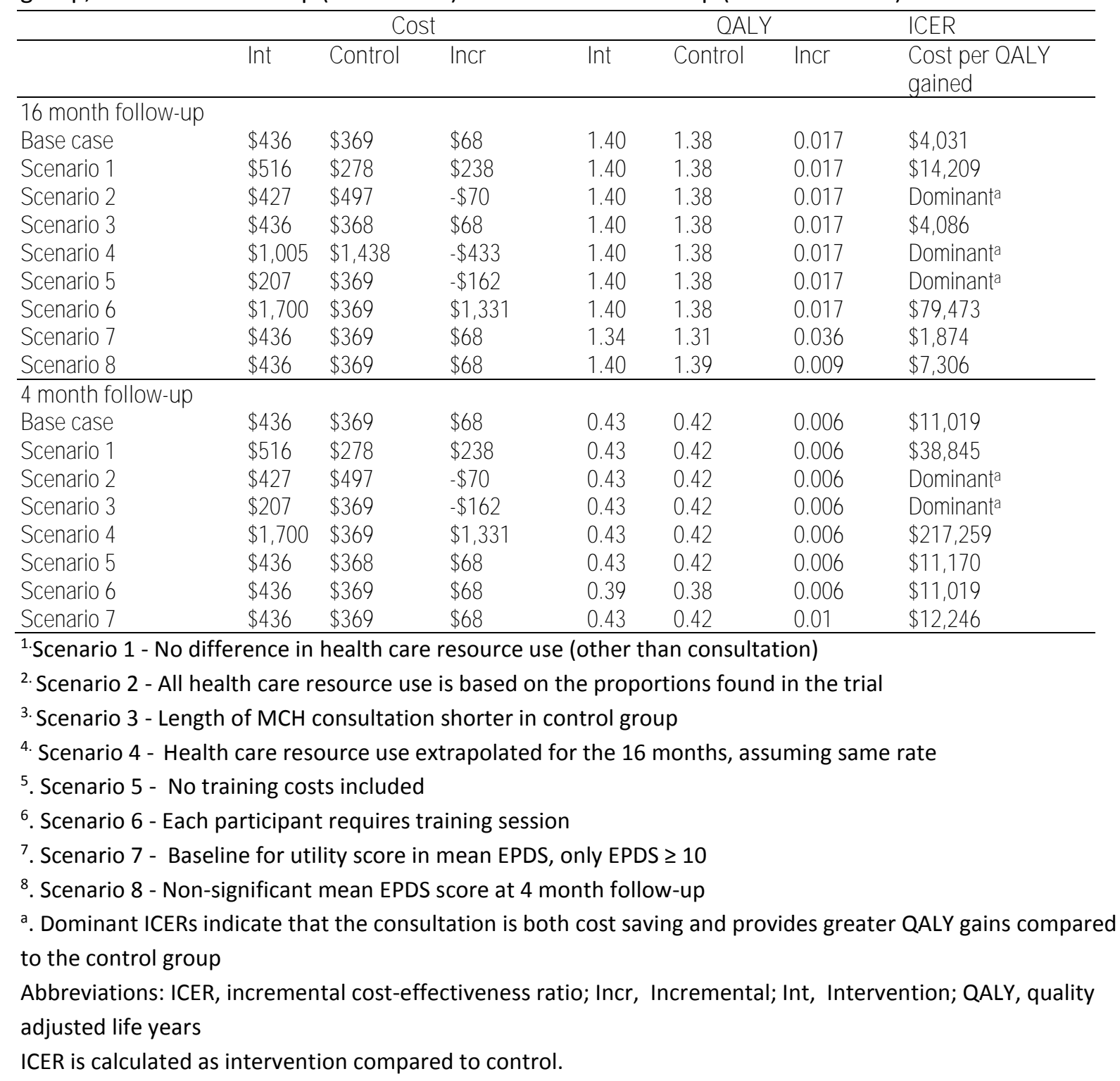

